\title{
Prevalencia de síntomas respiratorios indicativos de asma y asociación con contaminación atmosférica en preescolares de Bucaramanga, Colombia
}

\author{
Laura Andrea Rodríguez ${ }^{1}$, Juan José Rey ${ }^{1}$, Astrid Berena Herrera², Henry Castro ${ }^{3}$, \\ Jurg Niederbacher ${ }^{2,4}$, Lina María Vera², Luz Libia Cala ${ }^{2,4}$, Fabio Bolívar ${ }^{2,4}$ \\ 1 Observatorio de Salud Pública de Santander, Bucaramanga, Colombia \\ 2 Escuela de Medicina, Universidad Industrial de Santander, Bucaramanga, Colombia \\ 3 Corporación para la Defensa de la Meseta de Bucaramanga, Bucaramanga, Colombia \\ ${ }^{4}$ Instituto Neumológico del Oriente, Bucaramanga, Colombia
}

Introducción. Actualmente se sabe que el incremento de los niveles de contaminación atmosférica externa e interna se asocia con efectos nocivos para la salud, especialmente asma y otras alergias.

Objetivos. Determinar la prevalencia de síntomas respiratorios indicativos de asma en la población preescolar y compararla entre zonas con diferentes niveles de contaminación atmosférica en Bucaramanga, Colombia.

Materiales y métodos. Estudio observacional analítico de corte transversal que compara los síntomas respiratorios en menores de siete años de dos zonas de la ciudad con niveles diferentes de contaminación por material sólido en partículas y ozono. La morbilidad fue medida con un cuestionario sobre síntomas respiratorios indicativos de asma, validado al español.

Resultados. Se estudiaron 768 niños distribuidos de las zonas de alta y baja contaminación. El uso de cigarrillos $(27,5 \%)$ y aerosoles $(22,7 \%)$ se encontraron como las fuentes de contaminación más frecuentes dentro de las viviendas. La prevalencia de ruidos respiratorios a lo largo de la vida fue de $25,6 \%$ (IC95\% 23,2-29,8) y la de diagnóstico médico de asma fue de 8,4\% (IC95\% 6,2-11,5), sin diferencias significativas entre las zonas. El modelo final no mostró asociación entre la contaminación atmosférica y la presencia de síntomas respiratorios indicativos de asma. Por el contrario, la presencia de pisos de cemento y moho mostró una asociación significativa con estos síntomas.

Conclusiones. Es posible que en Bucaramanga los factores de contaminación domiciliaria puedan tener un mayor efecto sobre la presencia de síntomas respiratorios indicativos de asma en menores de siete años que los contaminantes externos.

Palabras clave: signos y síntomas respiratorios, asma/epidemiología, contaminación del aire, preescolar.

Respiratory symptoms associated with asthma prevalence and air pollution in preschool children

Introduction. Increases in the air pollution levels has well-documented harmful effects on human health, especially exacerbating problems of asthma and other allergies.

Objective. The prevalence of symptoms associated with asthma was determined in preschool populations in zones with differing levels of air pollution.

Materials and methods. A cross-sectional study was conducted in Bucaramanga, Colombia, comparing respiratory symptoms in 768 children under seven years in two urban zones with different pollution levels of particulate matter and ozone. Morbidity was evaluated for respiratory indicators of asthma by means of a questionnaire version previously validated in Spanish.

Results. The use of cigarettes (27.5\%) and aerosols (22.7\%) were the most frequent source of indoor air pollution. Wheezing prevalence was $25.6 \%(95 \% \mathrm{Cl} 23.2-29.8 \%)$ and diagnosis of asthma was $8.4 \%(95 \% \mathrm{Cl} 6.2-11.5 \%)$, with no differences between zones. The final multivariate model did not show an association between outdoor pollution and symptoms related with asthma. However, concrete flooring and presence of mold did show an association with these symptoms.

Conclusions. Indoor air quality rather than outdoor air pollution may play a more important role in producing respiratory symptoms related with asthma in preschool children in the city of Bucaramanga, Colombia.

Key words: signs and symptoms, respiratory; asthma/epidemiology, air pollution; child, preschool. 
Las acciones humanas han tenido un efecto perjudicial en la composición del aire, especialmente porque la quema de combustibles fósiles y otras actividades industriales han introducido contaminantes, incluidos el dióxido de azufre $\left(\mathrm{SO}_{2}\right)$, el monóxido de carbono (CO), los compuestos orgánicos volátiles, los óxidos de nitrógeno y partículas sólidas y líquidas, conocidas como material en partículas (1). El material en partículas es un contaminante crítico, de muy variable composición química, denominado primario ya que se emite directamente por fuentes naturales, humanas e industriales, que es vigilado mundialmente por la importancia que representan sus efectos sobre la salud humana. Este contaminante está compuesto principalmente por pequeñas partículas sólidas de polvo, humo y ceniza volátil, o líquidas formadas a partir de procesos de condensación de algunos gases presentes como niebla en la atmósfera (2).

En la actualidad, se sabe que el incremento de los niveles de contaminación atmosférica externa e interna se asocia con efectos nocivos para la salud, especialmente asma y otras alergias (3). La carga social y económica de la contaminación atmosférica es alta y se encuentra asociada con elevados gastos médicos debidos a morbilidad cardiovascular y respiratoria. Se estima que causa alrededor de 800.000 muertes prematuras por año en el mundo (4). La problemática de la contaminación atmosférica asociada a alteraciones de la salud entre la población, es un tema de alta relevancia entre las entidades ambientales y de salud a nivel mundial (5).

Sin embargo, la gran mayoría de los estudios desarrollados que pretenden encontrar una relación dosis-respuesta de poblaciones expuestas a ciertos contaminantes, han sido debatidos en el ámbito técnico, principalmente, por la ambigüedad en las mediciones de la exposición (1). Las investigaciones se han enfocado últimamente al estudio de la fracción respirable, menores de 10 $\mu \mathrm{m}$ y, más recientemente, menores de $2,5 \mu \mathrm{m}$.

En los niños, la exposición a partículas se ha asociado con un incremento en la frecuencia de signos y síntomas respiratorios como tos, dificultad

$\overline{\text { Correspondencia: }}$

Laura Andrea Rodríguez, Calle 49 № 27A-74, Bucaramanga, Colombia

Telefax: (057) 6434407

laurarovi78@gmail.com

Recibido: 11/02/09; aceptado:27/07/09 para respirar y dolor en el pecho, disminución del volumen espiratorio del primer segundo (forced expiratory volumen in 1 second, $\mathrm{FEV}_{1}$ ), de la capacidad vital forzada (forced expiratory vital capacity, FVC) y de la relación $\mathrm{FEV}_{1} / \mathrm{FVC}$, así como de las infecciones respiratorias y enfermedades respiratorias pulmonares (6).

En Colombia, el Estudio Nacional de Prevalencia de Asma estimó una proporción entre 8\% y 13\% en población infantil. Sin embargo, estudios locales como el realizado en Cali, han estimado prevalencias de asma hasta de $20 \%$ y rinitis alérgica de $18 \%$ en preescolares, especialmente de estratos socioeconómicos bajos (7).

En Santander, un análisis reciente de morbilidad basado en los Registros Individuales de Prestación de Servicios, estableció que el asma es la tercera causa de atención en consulta externa y la primera en urgencias entre la población pediátrica de los diferentes regímenes de aseguramiento (8).

El objetivo de este estudio fue describir algunos factores de contaminación en zonas definidas como de alta y baja contaminación de Bucaramanga, Colombia, y comparar la presencia de síntomas respiratorios agudos y crónicos indicativos de asma en la población pediátrica residente en dichas zonas.

\section{Materiales y métodos}

Se realizó un estudio observacional analítico de corte transversal, comparando dos zonas de la ciudad con niveles diferentes de contaminación según valores previos de material en partículas de la fracción respirable menor de $10 \mu \mathrm{m}$ (PM10) y ozono.

La selección de las zonas de monitorización se realizó entre mayo de 2006 y mayo de 2007, determinando con equipos de monitorización las zonas de la ciudad con mayores y menores registros de PM10. De esta forma, se seleccionó la zona de mayor registro, denominada de "alta" contaminación en el centro de la ciudad y la zona de menor registro, denominada de "baja" contaminación en la zona occidental de la ciudad $\left(89,56 \mu \mathrm{g} / \mathrm{m}^{3}\right.$, promedio en zona denominada de alta contaminación, y $40,08 \mu \mathrm{g} / \mathrm{m}^{3}$ en promedio en la zona que se denominó de baja contaminación, con una diferencia promedio de PM10 de 49,48 $\left.\mu \mathrm{g} / \mathrm{m}^{3}\right)$.

La población de estudio correspondió a los menores de siete años (predominantemente preescolares) que, por su condición, corresponden 
a los residentes más habituales y vulnerables en las zonas señaladas.

Se calculó un tamaño de muestra en el programa Epi-Info 6.04d, teniendo en cuenta un error tipo 1\% del $5 \%$, poder del $80 \%$, razón de expuestos y no expuestos de 1:1, prevalencia del factor de $18 \%$ en expuestos y $10 \%$ en no expuestos y un riesgo relativo esperado de 1,8 (odds ratio, $\mathrm{OR}=1,93$ ); se obtuvo un número mínimo de 319 niños en cada nivel de exposición, para un total de 638 niños. Se tuvo en cuenta un $20 \%$ de pérdidas en el seguimiento para un estudio posterior de cohorte, por lo cual se determinó una muestra total de 764 niños en las zonas de estudio.

La muestra se seleccionó de forma no probabilística, partiendo desde el sitio de monitorización ambiental hacia la periferia e incluyendo menores de siete años con residencia mayor de seis meses en el sector. Se excluyeron niños con enfermedades cardiacas o respiratorias crónicas o con alteración neurológica crónica (parálisis cerebral, trastorno de la deglución, etc.).

La morbilidad aguda se midió por medio del cuestionario International Study of Asthma and Allergies in Childhood (ISAAC) (9) de síntomas respiratorios indicativos de asma en su versión validada al español (10) y fue aplicado por fisioterapeutas bajo la coordinación de neumólogos pediatras, entre junio y julio de 2007, como línea de base de un estudio de seguimiento de síntomas respiratorios a un año.

Las variables sociodemográficas y las de presencia o ausencia de otros contaminantes en la vivienda, se obtuvieron mediante encuesta directa a los padres o cuidadores.

Se hizo un análisis descriptivo de los hallazgos por medio de medidas de tendencia central y variabilidad para las variables cuantitativas y proporciones con intervalos de confianza del 95\% para las variables discretas. El análisis bivariado y estratificado tuvo en cuenta como variable explicativa principal de los síntomas respiratorios, el tipo de zona (alta o baja contaminación).

Finalmente, se realizó un análisis multivariado con regresión de tipo binomial para ajustar el efecto de la contaminación por otros factores y se tuvo como variables dependientes de análisis la presencia de ruidos respiratorios alguna vez en la vida que, en el cuestionario ISAAC, se considera el signo más relacionado con el diagnóstico clínico de asma.
Para los análisis, se utilizó el programa Stata 9.0. El estudio respetó los principios de autonomía, confidencialidad y beneficencia, de acuerdo con las normas internacionales vigentes para investigación en seres humanos.

El protocolo de investigación fue aprobado por el Comité de Ética de la Facultad de Salud de la Universidad Industrial de Santander.

\section{Resultados}

Se estudiaron 768 niños distribuidos en las zonas de alta y baja contaminación seleccionadas (384 niños en cada zona). En el cuadro 1 se presentan algunas características sociodemográficas de la población de estudio.

El $84 \%$ de las encuestas fueron respondidas por los cuidadores habituales de los niños: la madre $(60,8 \%)$, la abuela $(14,8 \%)$ o el padre $(8,4 \%)$. La mitad de los niños (53\%) asistía regularmente a guarderías ubicadas, principalmente, dentro del barrio (65\%). En promedio, la composición familiar de los niños encuestados tenía seis integrantes, de los cuales, dos eran niños menores de 18 años.

Las viviendas estaban ubicadas en barrios de estratos socioeconómicos 2 y 3 , y su estructura se caracterizaba por tener entre tres y cinco cuartos (73\%), techos de placa (61\%) y pisos de baldosín (95\%). El tabaquismo $(27,5 \%)$ y los aerosoles $(22,7 \%)$ se encontraron como las fuentes de contaminación más frecuentes dentro de las viviendas, siendo más frecuentes en la zona de baja contaminación (cuadro 2). El combustible para cocinar era predominantemente el gas (95\%) y el uso de leña sólo se encontró en $0,5 \%$ de las viviendas. La mitad de las viviendas reportaron la presencia de dos contaminantes internos y en $10 \%$ se documentaron hasta cuatro.

Por su parte, la presencia de cucarachas y la de animales domésticos (perros, gatos y aves) fueron otros factores ambientales encontrados con bastante frecuencia dentro de las viviendas (54\% y $35 \%$, respectivamente).

En las zonas de estudio, se encontraron posibles focos de contaminación atmosférica alrededor de las viviendas representados, principalmente, por talleres de zapatería en la zona de baja contaminación (96\%) y, en la zona de alta contaminación, por estos talleres junto con otros dedicados a carpintería/ tapicería $(84,6 \%)$ y ornamentación $(70,3 \%)$.

Como se muestra en el cuadro 2, los habitantes de las dos zonas perciben la presencia de alto 
Cuadro 1. Características sociodemográficas de la población, Bucaramanga, Colombia, 2007.

\begin{tabular}{|c|c|c|c|c|c|c|}
\hline \multirow[t]{2}{*}{ Variable } & \multicolumn{2}{|c|}{$\begin{array}{l}\text { Zona de alta } \\
\text { contaminación } \\
\quad(\mathrm{N}=384)\end{array}$} & \multicolumn{2}{|c|}{$\begin{array}{c}\text { Zona de baja } \\
\text { contaminación } \\
\quad(\mathrm{N}=384)\end{array}$} & \multicolumn{2}{|c|}{$\begin{array}{l}\text { Población total } \\
\qquad(\mathrm{N}=768)\end{array}$} \\
\hline & $\mathbf{n}$ & $\%$ & $\mathbf{n}$ & $\%$ & $\mathbf{n}$ & $\%$ \\
\hline \multicolumn{7}{|l|}{ Sexo } \\
\hline Femenino & 170 & 44,3 & 168 & 43,8 & 338 & 44,0 \\
\hline Masculino & 214 & 55,7 & 216 & 56,3 & 430 & 56,0 \\
\hline Edad promedio (meses) & 43,3 & 42,3 & 42,8 & & & \\
\hline \multicolumn{7}{|l|}{ Tiempo de residencia en el sector (meses) } \\
\hline 6 a 12 & 86 & 22,4 & 76 & 19,8 & 162 & 21,1 \\
\hline 13 a 18 & 18 & 4,7 & 26 & 6,8 & 44 & 5,7 \\
\hline 19 a 24 & 5 & 1,3 & 14 & 3,6 & 19 & 2,5 \\
\hline$>24$ & 275 & 71,6 & 268 & 69,8 & 543 & 70,7 \\
\hline \multicolumn{7}{|l|}{ Asistencia a guardería } \\
\hline Sí & 203 & 52,9 & 203 & 52,9 & 406 & 52,9 \\
\hline No & 178 & 46,4 & 172 & 44,8 & 350 & 45,6 \\
\hline Sin dato & 3 & 0,8 & 9 & 2,3 & 12 & 1,6 \\
\hline \multicolumn{3}{|l|}{ Régimen de seguridad social en salud } & & 0,0 & & 0,0 \\
\hline Contributivo & 278 & 72,4 & 257 & 66,9 & 535 & 69,7 \\
\hline Subsidiado & 68 & 17,7 & 91 & 23,7 & 159 & 20,7 \\
\hline Vinculado & 37 & 9,6 & 34 & 8,9 & 71 & 9,2 \\
\hline No sabe & 1 & 0,3 & 2 & 0,5 & 3 & 0,4 \\
\hline
\end{tabular}

Cuadro 2. Contaminantes dentro y alrededor de las viviendas de las zonas de estudio, Bucaramanga, Colombia, 2007.

\begin{tabular}{|c|c|c|c|c|c|c|}
\hline \multirow[t]{2}{*}{ Variable } & \multicolumn{2}{|c|}{$\begin{array}{l}\text { Zona de alta } \\
\text { contaminación } \\
\quad(\mathrm{N}=384)\end{array}$} & \multicolumn{2}{|c|}{$\begin{array}{l}\text { Zona de baja } \\
\text { contaminación } \\
\quad(\mathrm{N}=384)\end{array}$} & \multicolumn{2}{|c|}{$\begin{array}{l}\text { Población total } \\
\qquad(\mathrm{N}=768)\end{array}$} \\
\hline & $\mathbf{n}$ & $\%$ & $\mathbf{n}$ & $\%$ & $\mathbf{n}$ & $\%$ \\
\hline Tabaco & 6 & 1,6 & 6 & 1,6 & 12 & 1,6 \\
\hline Aerosoles & 73 & 19,0 & 101 & 26,3 & 174 & 22,7 \\
\hline Leña & 1 & 0,3 & 5 & 1,3 & 6 & 0,8 \\
\hline Carbón & 1 & 0,3 & 3 & 0,8 & 4 & 0,5 \\
\hline \multicolumn{7}{|c|}{$\begin{array}{l}\text { Otros factores ambientales presentes dentro de } \\
\text { la vivienda }\end{array}$} \\
\hline Moho y hongos & 43 & 11,2 & 160 & 41,7 & 203 & 26,4 \\
\hline Polen & 63 & 16,4 & 120 & 31,3 & 183 & 23,8 \\
\hline Cucarachas & 216 & 56,3 & 200 & 52,1 & 416 & 54,2 \\
\hline Gato & 32 & 8,3 & 34 & 8,9 & 66 & 8,6 \\
\hline Perro & 98 & 25,5 & 176 & 45,8 & 274 & 35,7 \\
\hline Aves & 106 & 27,6 & 163 & 42,4 & 269 & 35,0 \\
\hline Roedores & 122 & 31,8 & 124 & 32,3 & 246 & 32,0 \\
\hline \multicolumn{7}{|c|}{ Contaminantes alrededor de la vivienda } \\
\hline Hornos & 67 & 17,4 & 22 & 5,7 & 89 & 11,6 \\
\hline Ornamentación & 270 & 70,3 & 135 & 35,2 & 405 & 52,7 \\
\hline Zapatería & 340 & 88,5 & 371 & 96,6 & 711 & 92,6 \\
\hline Basurero/escombrero & 133 & 34,6 & 81 & 21,1 & 214 & 27,9 \\
\hline Carpintería/tapicería & 325 & 84,6 & 185 & 48,2 & 510 & 66,4 \\
\hline Alto flujo vehicular & 359 & 93,5 & 372 & 96,9 & 731 & 95,2 \\
\hline Construcciones & 212 & 55,2 & 188 & 49,0 & 400 & 52,1 \\
\hline
\end{tabular}


flujo vehicular alrededor de sus viviendas, a pesar de que el tránsito vehicular en la zona de baja contaminación tiene una única vía principal con transporte público restringido a las rutas que ingresan al barrio, contrario a la zona de alta contaminación que es atravesada por una de las vías de más alto flujo vehicular de la ciudad.

La prevalencia de ruidos respiratorios a lo largo de la vida fue de $25,6 \%$ (IC95\% 23,2-29,8) y la de diagnóstico médico de asma fue de 8,4\% (IC95\% $6,2-11,5)$. Teniendo en cuenta como variables dependientes la presencia de síntomas y los diagnósticos respiratorios, el análisis bivariado mostró que solamente la de rinitis tuvo asociación con la zona de residencia según el nivel de contaminación (prevalencia relativa, $P R=1,44$ ) (IC95\% 1,17-1,78) (cuadro 3).

El análisis multivariado evaluó el efecto del nivel de contaminación con respecto a la presencia de ruidos respiratorios alguna vez en la vida, que se considera el signo respiratorio de asma que más se relaciona con su diagnóstico clínico dentro del cuestionario ISAAC. Luego de ajustar por el efecto de posibles variables de confusión, el modelo final no mostró asociación entre estas variables y la presencia de ruidos respiratorios alguna vez en la vida.

Por el contrario, la presencia de pisos de cemento y moho mostró una asociación significativa con este signo (cuadro 4).

\section{Discusión}

El presente estudio muestra que no hay asociación entre la contaminación extradomiciliaria y la presencia de síntomas respiratorios indicativos de asma, en una población de preescolares residentes en dos zonas con diferentes niveles de contaminación de Bucaramanga.

La medición de la exposición (contaminación atmosférica extradomiciliaria) y la morbilidad respiratoria tuvieron en cuenta instrumentos y parámetros aceptados y utilizados ampliamente en este tipo de estudios (11-13). Para medir la exposición, se usó la captación y cuantificación de material en partículas de $10 \mu \mathrm{m}$ (PM10), por personal entrenado de la institución de competencia ambiental de la ciudad, y tuvo un período de delimitación de las zonas y ajuste de instrumentos de un año antes del inicio de la encuesta que validó la diferencia de niveles de PM10 entre las zonas de comparación.

La zona de alta contaminación, a diferencia de la zona denominada de baja contaminación, mantuvo niveles de promedio anual superiores a $80 \mu \mathrm{g} / \mathrm{m}^{3}$ y en varias ocasiones sobrepasó la norma local máxima diaria de $134 \mu \mathrm{g} / \mathrm{m}^{3}$, según la legislación colombiana (14).

En cuanto a la medición de la morbilidad respiratoria, se usó el cuestionario validado al español del estudio ISAAC (10), por medio de encuestadoras profesionales de salud con experiencia en manejo clínico de síntomas respiratorios. Este cuestionario ha sido usado en diferentes países, incluso de habla hispana, lo cual proporciona un alto grado de confiabilidad y permite comparar nuestros resultados con otros estudios.

El cuestionario de síntomas respiratorios se aplicó a padres o cuidadores, por lo que existe la probabilidad de un sesgo de memoria; sin embargo, la encuesta fue aplicada en las mismas condiciones y por las mismas encuestadoras entrenadas en las dos zonas del estudio, por lo que, de existir éste, no tendría diferencias entre los grupos.

De igual forma, existe la posibilidad de un sesgo de información (del entrevistado), por el conocimiento previo a la encuesta del objetivo del estudio. En este caso, se esperaría una sobreestimación de la prevalencia de síntomas reportados; sin embargo, las prevalencias obtenidas concuerdan con las encontradas en un estudio previo en la ciudad y en otras ciudades colombianas (15) y no fueron diferenciales entre las dos zonas de comparación.

La prevalencia acumulada de signos y síntomas indicativos de asma (haber presentado alguna vez en la vida ruidos en el pecho al respirar) fue de $25,6 \%$ y la de asma diagnosticada por médico fue de $8,4 \%$, similares a las encontradas por Dennis et al. (15) que fueron de $28,1 \%$ y $8,4 \%$, respectivamente, en el grupo de 5 a 11 años. Se han encontrado resultados de prevalencia similares, también, en escolares de ciudades de Perú (16).

Nuestros resultados contrastan con otros estudios que muestran asociación de la contaminación medida por PM10 con asma o síntomas respiratorios $(17,18)$.

Un metanálisis de los efectos agudos de la contaminación sobre la salud de la población, mostró asociación con eventos agudos de mortalidad, hospitalizaciones, consultas de urgencias y algunos síntomas respiratorios como tos y ataques de asma en poblaciones susceptibles; sin embargo, los estudios no son específicos en población general preescolar (19). 
Cuadro 3. Síntomas y diagnósticos de morbilidad respiratoria en población pediátrica según zonas de contaminación atmosférica, Bucaramanga, Colombia, 2007.

\begin{tabular}{|c|c|c|c|c|c|c|}
\hline \multirow[t]{2}{*}{ Variable } & \multicolumn{2}{|c|}{$\begin{array}{l}\text { Zona de alta } \\
\text { contaminación } \\
(\mathrm{N}=384)\end{array}$} & \multicolumn{2}{|c|}{$\begin{array}{l}\text { Zona de baja } \\
\text { contaminación } \\
\quad(\mathrm{N}=384)\end{array}$} & \multirow[t]{2}{*}{$\begin{array}{l}\text { Prevalencia } \\
\text { relativa }\end{array}$} & \multirow[t]{2}{*}{ IC 95\% } \\
\hline & $\mathrm{n}(\%)$ & IC $95 \%$ & n (\%) & IC 95\% & & \\
\hline \multicolumn{7}{|l|}{$\begin{array}{l}\text { Presencia de síntomas } \\
\text { respiratorios en el último mes }\end{array}$} \\
\hline Resfriado & $183(47,7)$ & $42,6-58,8$ & $210(54,7)$ & $49,6-59,7$ & 0,87 & $0,75-1,00$ \\
\hline Rinitis & $149(38,8)$ & $33,9-43,9$ & $103(26,8)$ & $22,5-31,6$ & 1,44 & $1,17-1,78$ \\
\hline Lagrimeo & $48(12,5)$ & $9,4-16,3$ & $60(15,6)$ & $12,2-19,7$ & 0,80 & $0,56-1,13$ \\
\hline Amigdalitis & $65(16,9)$ & $13,4-21,1$ & $86(22,4)$ & $18,4-27,0$ & 1,30 & $0,73-2,28$ \\
\hline Otitis & $39(10,2)$ & $7,4-13,7$ & $75(19,5)$ & $15,8-23,9$ & 0,33 & $0,15-0,69$ \\
\hline Irritación ocular sola & $25(6,5)$ & $4,3-9,6$ & $59(15,4)$ & $12,0-19,5$ & 0,42 & $0,27-0,66$ \\
\hline $\begin{array}{l}\text { Irritación ocular asociada } \\
\text { a enfermedad respiratoria }\end{array}$ & $55(14,3)$ & $1,1-18,3$ & $86(22,4)$ & $18,4-27,0$ & 0,63 & $0,47-0,86$ \\
\hline Necesidad de consulta médica & $115(29,9)$ & $25,5-34,8$ & $123(32)$ & $27,4-37,0$ & 0,93 & $0,65-1,15$ \\
\hline \multicolumn{7}{|l|}{$\begin{array}{l}\text { Presencia de síntomas relacionados } \\
\text { con asma en el último año }\end{array}$} \\
\hline $\begin{array}{l}\text { Episodio de sibilancias alguna vez } \\
\text { en la vida }\end{array}$ & $86(22,4)$ & $18,4-27,0$ & $111(28,9)$ & $24,5-33,9$ & 0,77 & $0,60-0,98$ \\
\hline $\begin{array}{l}\text { Historia de diagnóstico médico de } \\
\text { asma bronquial }\end{array}$ & $24(6,3)$ & $4,1-9,3$ & $41(10,7)$ & $7,9-14,3$ & 0,58 & $0,36-0,94$ \\
\hline Episodio de sibilancias & $52(13,5)$ & $10,4-17,5$ & $55(14,3)$ & $11,1-18,4$ & 0,94 & $0,66-1,03$ \\
\hline Sibilancias con despertar nocturno & $36(9,4)$ & $6,8-12,9$ & $33(8,6)$ & $6,1-12,0$ & 1,08 & $0,69-1,70$ \\
\hline $\begin{array}{l}\text { Sibilancias o asfixia que interrumpen } \\
\text { el habla }\end{array}$ & $19(4,9)$ & $3,1-7,8$ & $15(3,9)$ & $2,3-6,5$ & 1,26 & $0,65-2,44$ \\
\hline $\begin{array}{l}\text { Necesidad de consulta de urgencias } \\
\text { u hospitalización }\end{array}$ & $26(6,8)$ & $4,3-9,6$ & $32(8,3)$ & $5,9-11,7$ & 0,81 & $0,49-1,33$ \\
\hline Sibilancias relacionadas con ejercicio & $20(5,2)$ & $3,3-8,1$ & $14(3,6)$ & $2,1-6,2$ & 1,42 & $0,73-2,77$ \\
\hline Sibilancias durante episodios de gripa & $40(10,4)$ & $7,6-14$ & $50(13,0)$ & $9,9-16,9$ & 0,79 & $0,54-1,18$ \\
\hline $\begin{array}{l}\text { Ataques de tos seca nocturnos en } \\
\text { ausencia de gripa o infección respiratoria }\end{array}$ & $119(31,0)$ & $26,4-35,9$ & $136(35,4)$ & $30,8-40,7$ & 0,87 & $0,71-1,06$ \\
\hline \multicolumn{7}{|l|}{$\begin{array}{l}\text { Diagnósticos médicos realizados } \\
\text { en el último mes }\end{array}$} \\
\hline Otitis & $9(2,3)$ & $1,1-4,6$ & $27(7,0)$ & $4,8-10,2$ & 0,33 & $0,15-0,70$ \\
\hline Sinusitis & $2(0,5)$ & $0,1-21$ & $2(0,5)$ & $0,1-2,1$ & 1 & $0,14-7,06$ \\
\hline Amigdalitis & $26(6,8)$ & $4,6-9,9$ & $20(5,2)$ & $3,3-8,1$ & 1,3 & $0,73-2,28$ \\
\hline Infección respiratoria aguda (gripa) & $76(19,8)$ & $16,0-24,2$ & $78(20,3)$ & $16,5-24,8$ & 0,97 & $0,73-1,29$ \\
\hline Crup & $2(0,5)$ & $0,1-2,1$ & $0(0,0)$ & $0,0-0,0$ & 0 & \\
\hline Asma & $8(2,1)$ & $1,0-4,2$ & $8(2,1)$ & $1,0-4,2$ & 1 & $0,38-2,63$ \\
\hline Neumonía & $0(0,0)$ & $0,0-0,0$ & $3(0,8)$ & $0,2-2,5$ & 0 & \\
\hline Bronquitis & $4(1,0)$ & $0,3-2,8$ & $6(1,6)$ & $0,6-3,5$ & 0,66 & $0,19-2,34$ \\
\hline Bronconeumonía & $3(0,8)$ & $0,2-2,5$ & $1(0,3)$ & $0,0-1,7$ & 3 & $0,31-28,71$ \\
\hline
\end{tabular}

Cuadro 4. Modelo ajustado multivariado binomial de síntomas respiratorios indicativos de asma.

\begin{tabular}{lcc}
\hline Variable & Prevalencia relativa & Intervalo de confianza 95\% \\
\hline Zona de alta contaminación & 1,00 & $0,76-1,32$ \\
Sexo masculino & 1,35 & $1,05-1,72$ \\
Edad en meses & 1,00 & $1,00-1,00$ \\
Piso de cemento en la vivienda & 1,50 & $1,15-1,96$ \\
Presencia de moho & 1,12 & $0,86-1,47$ \\
Enfermedad respiratoria en el último año & 2,21 & $1,39-3,52$ \\
\hline
\end{tabular}

En Chile, Zamorano et al. (20) estudiaron el efecto de la contaminación ambiental sobre la presentación de bronquiolitis aguda en menores de un año y tampoco encontraron asociación significativa con esta variable; la exposición a tabaco y la edad temprana fueron los factores más relacionados con esta enfermedad.

Hallazgos epidemiológicos recientes sustentan el efecto de la contaminación externa medida con PM10 sobre la mortalidad y morbilidad respiratoria 
y cardiovascular aguda; sin embargo, en la mayoría de áreas urbanas la asociación es más fuerte para las partículas más finas (material en partículas de $2,5 \mu \mathrm{m}) \mathrm{y}$, de forma similar a nuestros resultados, en otros estudios no parece tener una asociación fuerte con los niveles de PM10, especialmente en niños $(21,22)$.

Otra posible razón para explicar la ausencia de asociación entre las variables de interés, pudo haber sido que la diferencia de niveles de contaminación (PM10) entre las zonas de estudio no fuera lo suficientemente grande y la zona indicada como de alta contaminación no tuviera niveles lo suficientemente altos para causar un impacto en la morbilidad respiratoria. En este sentido, es posible que el estudio haya podido incurrir en un error tipo II debido a que la diferencia en morbilidad pudiera haber sido tan pequeña a estos gradientes de nivel de PM10, que no haya podido demostrarse con la muestra seleccionada.

Sin embargo, las mediciones de PM10 durante el año de monitorización previa y durante el tiempo de la realización de la encuesta siempre mantuvieron diferencias mínimas de 30 puntos, manteniendo la zona baja niveles considerados buenos y, la zona alta, niveles considerados regulares y malos, según la norma colombiana ajustada a condiciones regionales (14), por lo cual, a diferencia del estudio similar realizado en Bogotá (23), no parece ser esa la razón de la ausencia de asociación entre PM10 y la presencia de síntomas respiratorios.

Por otra parte, entre las otras variables estudiadas se encontró que la presencia de piso de cemento y mohos dentro de la vivienda eran factores ambientales domiciliarios que sí mostraron asociación significativa de forma independiente con la presencia de ruidos respiratorios. Estos hallazgos se relacionan con un estudio local reciente que analizó las partículas aerotransportadas en la ciudad y demostró que los hongos eran las más frecuentemente encontradas (85,34\%) (24).

En este tema, diversos estudios han demostrado la asociación de contaminantes domiciliares, como hongos, ácaros, cucarachas, combustibles de biomasa y humo de cigarrillo, entre otros, con la presencia de morbilidad respiratoria, especialmente en niños, que permanecen la mayor parte del tiempo en sus casas (25-32).

Estos hallazgos sugieren la hipótesis de que, en Bucaramanga, los factores de contaminación intradomiciliaria pueden tener un mayor efecto sobre la presencia de síntomas respiratorios indicativos de asma en menores de siete años que los contaminantes externos, por lo cual se recomienda iniciar un estudio de este tipo de factores con el fin de determinar los más influyentes en eventos respiratorios agudos.

De igual forma, se recomienda continuar el estudio de los factores contaminantes externos que pueden estar afectados por variaciones estacionales anuales de humedad y temperatura, y que no fueron analizados en este trabajo, por tratarse de un estudio de corte transversal.

\section{Conflicto de intereses}

Los autores declaran no tener conflicto de intereses.

\section{Financiación}

Este estudio fue financiado por Colciencias (Proyecto No. 651734519008), el Observatorio de Salud Púbica de Santander, la Corporación para la Defensa de la Meseta de Bucaramanga, el Instituto Neumológico del Oriente, la Universidad Industrial de Santander y la Secretaría de Salud de Santander.

\section{Referencias}

1. Environmental Protection Agency. Particulate Matter Research Program: five years of progress. Washington, D.C.: EPA; 2004.

2. OMS, OPS. Área de desarrollo sostenible y salud ambiental. Control de la contaminación del aire. Fecha de consulta: noviembre, 2007. Disponible en:http://www.cepis.ops-oms.org

3. Ballester F. La evaluación del impacto en salud de la contaminación atmosférica. Rev Salud Ambiente. 2003;3:102-7.

4. Curtis L, Rea W, Smith-Willis P, Fenyves F, Pan Y. Adverse health effects of outdoor air pollutants. Environ Int. 2006;32:815-30

5. Vargas F. La contaminación ambiental como factor determinante de la salud. Rev Esp Salud Pública. 2005:79:117-27.

6. Télles M, Romieu I, Polo M, Ruiz S, Meneses F, Hernández M. Efecto de la contaminación ambiental sobre las consultas por infecciones respiratorias en niños de la ciudad de México. Salud Pública Mex. 1997;39:513-22.

7. Arévalo M, Reyes M, Victoria I, Villegas A, Badiel M, Barrera S. Asma y rinitis alérgica en preescolares de Cali. Colombia Médica. 2003;34:4-8.

8. Observatorio de Salud Pública de Santander. Indicadores de morbilidad basados en el Registro Individual de Prestación de Servicios RIPS. Bucaramanga: Secretaría de Salud de Santander; 2006.

9. Asher M, Keil U, Anderson H, Beasley R, Crane J, Martínez F, et al. International study of asthma and allergies 
in childhood (ISAAC): rationale and methods. Eur Respir J. 1995;8:483-91.

10. Mata C, Fernández-Benítez M, Pérez Miranda M, Grima F. Validation of the Spanish version of the phase III ISAAC questionnaire on asthma. J Invest Allergol Clin Immunol. 2005;15:201-10.

11. Aranguez E, Ordoñes JM, Serrano J, Aragonés N, Fernández-Patier R, Gandarillas A, et al. Contaminantes atmosféricos y su vigilancia. Rev Esp Salud Pública. 1999;73:123-32.

12. Daston G, Faustman E, Ginsberg G, Fenner P, Olin S, Sonawane B, et al. A framework for assesing risk to children from exposure to enviromental agents. Environ Health Perspect. 2004;112:238-56.

13. Landrigan P, Kimmel C, Correa A, Eskenazi B. Children's health and the environment: public health issues and challenges for risk assessment. Environ Health Perspect. 2004;112:257-65.

14. Ministerio de Ambiente, Vivienda y Desarrollo Territorial. Resolución 601 del 4 de abril de 2006, por la cual se establece la Norma de Calidad del Aire o Nivel de Inmisión, para todo el territorio nacional en condiciones de referencia. Bogotá, D.C.: Ministerio de Ambiente, Vivienda y Desarrollo Territorial; 2006.

15. Dennis R, Caraballo L, García E, Caballera A, Aristizábal G, Córdoba H, et al. Asthma and other allergic conditions in Colombia: a study in 6 cities. Ann Allergy Asthma Inmunol. 2004;93:568-74.

16. Chiarella P, Vargas R, Vesga L, Navarro A, Martínez R, Gutierrez M, et al. Prevalencia de síntomas respiratorios compatibles con asma en niños escolares de 13 y 14 años de San Martín de Porras (Lima) y de Huancayo. Enferm Torax. 2004;48:50-8.

17. Romero M, Mas $\mathbf{P}$, Lacasaña $\mathbf{M}$, Téllez M, Aguilar J, Romieu I. Contaminación atmosférica, asma bronquial e infecciones respiratorias agudas en menores de edad, de La Habana. Salud Pública Mex. 2004;46:222-33.

18. Romeo E, De Sario M, Forastiere F, Compagnucci P, Bergamashi A, Perucci CA. PM10 exposure and asthma exacerbations in pediatric age: a metanalysis of panel and time series studies. Epidemiol Prev. 2006:40:245-54.

19. Rosales J, Torres V, Olaiz G, Borja V. Los efectos agudos de la contaminación del aire en la salud de la población: evidencia de estudios epidemiológicos. Salud Pública Mex. 2001;43:544-55.
20. Zamorano A, Márquez S, Aránguiz J, Bedregal $\mathbf{P}$, Sánchez I. Relación entre bronquiolitis aguda con factores climáticos y contaminación ambiental. Rev Med Chile. 2003; 131:1117-22.

21. Brunekreef B, Forsberg B. Epidemiological evidence of effects of coarse airborne particles on health. Eur Respir J. 2005;26:309-18.

22. Ward DJ, Ayres JG. Particulate air pollution and panel studies in children: a systematic review. Occup Environ Med. 2004;61:e13.

23. Solarte PI, Caicedo M, Restrepo S. Contaminación atmosférica y enfermedad respiratoria en niños menores de 14 años en Bogotá. Revista Médica Sánitas. 2002;5.

24. Cabrales C, García HE, Ramírez G. Estudio aerobiológico en la ciudad de Bucaramanga. Revista de alergia, asma e inmunología. Fecha de consulta: enero de 2009. Disponible en: http://encolombia.com/medicina/alergia/alergia12203estudio.htm.

25. Stark P, Celedón J, Chew G, Ryan L, Burge H, Muilenberg M. Fungal levels in the home and allergic rhinitis by 5 years of age. Environ Health Perspect. 2005;113:1405-9.

26. Dimitroulopolou C, Ashmore M, Hill M, Byrne M, Kinnersley R. INDAIR: A probabilistic model of indoor air pollution in UK homes. Atmos Environ. 2006;40:6362-79.

27. Dales R, Liu L, Wheeler A, Gilbert N. Quality of indoor residential air and health. CMAJ. 2008;179:147-52.

28. Franklin P. Indoor air quality and respiratory health of children. Paediatr Respir Rev. 2007;8:281-6.

29. Alvis N, De la Hoz F. Contaminación del aire domiciliario y enfermedades respiratorias (infección respiratoria aguda baja, EPOC, cáncer de pulmón y asma): evidencias de asociación. Rev Fac Med Unal. 2008;56:54-64.

30. Antova T, Pattenden S, Brunekreef B, Heinrich J, Rudnai P, Forastiere F, et al. Exposure to indoor mold and children's respiratory health in the PATY study. J Epidemiol Community Health. 2008;62:708-14.

31. Salo P, Xia J, Johnson A, Li Y, Avol E, Gong J, et al. Indoor alergens, asthma, and asthma related symptoms among adolescents in Wuhan, China. Ann Epidemiol. 2004;14:543-50.

32. Rivas E, Barrios S, Dorner A, Osorio X. Fuentes de contaminación intradomiciliaria y enfermedad respiratoria en jardines infantiles y salas cuna de Temuco y Padre Las Casas, Chile. Rev Med Chile. 2008;136:767-74. 